\title{
AMCoR
}

Asahikawa Medical University Repository http://amcor.asahikawa-med.ac.jp/

Digestive Endoscopy (2011) 23(4):319-323.

WATARI, JIRO ; MORITA, TSUYOSHI ; SAKURAI, JUN ; YAMASAKI, TAKAHISA : OKUGAWA, TAKUYA ;

TOYOSHIMA, FUMIHIKO ; KONDO, TAKASHI ; TANAKA, JUNJI ; TOMITA, TOSHIHIKO ; KIM, YONGMIN ; OSHIMA, TADAYUKI ; FUKUI, HIROKAZU ; HORI, KAZUTOSHI ; MORIICHI, KENTARO ; TANABE, HIROKI ; FUJIYA, MIKIHIRO ; KOHGO, YUTAKA ; OKU, JUNSUKE ; MATSUMOTO, TAKAYUKI ; MIWA, HIROTO 


\section{Endoscopically treated Cronkhite-Canada syndrome associated with minute intramucosal gastric cancer: an analysis of molecular pathology}

A brief title: Cronkhite-Canada syndrome associated with gastric cancer

Jiro Watari, ${ }^{1}$ Tsuyoshi Morita, ${ }^{1}$ Jun Sakurai, ${ }^{1}$, Takahisa Yamasaki, ${ }^{1}$ Takuya Okugawa, ${ }^{1}$ Fumihiko Toyoshima, ${ }^{1}$ Takashi Kondo, ${ }^{1}$ Junji Tanaka, ${ }^{1}$ Toshihiko Tomita, ${ }^{1}$ Yongmin Kim, ${ }^{1}$ Tadayuki Oshima, ${ }^{1}$ Hirokazu Fukui, ${ }^{1}$ Kazutoshi Hori, ${ }^{1}$ Kentaro Moriichi, ${ }^{2}$ Hiroki Tanabe,${ }^{2}$ Mikihiro Fujiya, ${ }^{2}$ Yutaka Kohgo, ${ }^{2}$ Junsuke Oku, ${ }^{3}$ Takayuki Matsumoto, ${ }^{3}$ Hiroto Miwa ${ }^{1}$

${ }^{1}$ Division of Upper Gastroenterology, ${ }^{3}$ Division of Lower Gastroenterology, Department of Internal Medicine, Hyogo College of Medicine, Nishinomiya, Japan; ${ }^{2}$ Division of Gastroenterology and Hematology/Oncology, Department of Medicine, Asahikawa Medical College, Asahikawa, Japan;

Key words: Cronkhite-Canada syndrome, Gastric cancer, Narrow band imaging, Endoscopic treatment, Molecular pathology

All authors have read and approved the final manuscript.

Disclosure: None of the authors have a conflict of interest. Moreover, we have no grants or financial support for this work. Written informed consent was obtained from the patient beforehand. 
Watari et al. 2

Address Correspondence to: Jiro Watari, MD

Division of Upper Gastroenterology,

Department of Internal Medicine,

Hyogo College of Medicine,

1-1, Mukogawa-cho, Nishinomiya, Hyogo 663-8501, Japan

Tel: +81-798-45-6662, Fax: +81-798-45-6661

E-mail: watarij@hyo-med.ac.jp 


\section{Abstract}

There has been no report of CCS associated gastric cancer resected by endoscopy because it is very difficult to identify small cancers, which are candidates for endoscopic resection. We report a case of CCS with gastric cancer treated by endoscopic submucosal dissection (ESD), and we evaluate the molecular pathological analysis of malignant transformation in patients with CCS. A 74-year-old man had an advanced rectal cancer and gastrointestinal polyposis after presenting with hypoproteinemia, partial hair loss and atrophic nails as well as hyperpigmentation on the hands, and was diagnosed as having CCS. On upper endoscopy, a 7-mm discolored polyp with an irregular microvascular pattern revealed by magnified narrow band imaging (NBI) was identified in gastric diffuse CCS polyposis. This lesion was treated by ESD and diagnosed as a flat, elevated-type, mucosal well-differentiated tubular adenocarcinoma without lymphatic or venous infiltration, and with tumor-free margins. Microsatellite instability was detected in both the cancer and the surrounding CCS polyps. Mucin-histochemical analysis of the cancer area showed the complete intestinal type, thus may be differentiate the CCS polyps from that of the common gastric hyperplastic polyps. This case illustrates that a clue to detecting small cancers may be to look for the discolored lesion among reddish CCS polyposis and thereafter to observe the irregular vascular pattern by NBI endoscopy. From the viewpoint of genetic alterations, patients with CCS polyps are considered to be at high risk for developing gastric cancer, and therefore careful follow-up examinations are necessary for the early detection of malignancies. 


\section{Introduction}

Cronkhite-Canada syndrome (CCS) is a rare hyperplastic gastrointestinal (GI) polyposis syndrome manifestied by various GI symptoms, alopecia, and onychodystrophy [1]. Although the syndrome’s etiology remains unclear, a significant number of patients with CCS with gastric or colorectal malignancy have been reported [2,3]. Interestingly, all cases of CCS associated with gastric cancer reported previously came from Japan [4]. Furthermore, all of them were surgically treated, but no case resected by endoscopy was seen. One probable reason for this is that it is very difficult to identify small cancers, which are candidates for endoscopic resection, in CCS polyposis. We herein report a rare case of CCS complicated by small gastric cancer and treated by endoscopic resection. Additionally, we evaluate the chronology of genetic instability and the cellular phenotype of this cancer and the background polyps.

\section{Case report}

A 74-year-old man was transported by ambulance to our hospital with impaired consciousness and tetraplegia in October 2009. He reported suffering from watery diarrhea for 1 month and deterioration in his general condition with loss of body weight. His past history was unremarkable, including no family history of malignancy or GI polyposis. Physical examination revealed a well-developed man with partial hair loss and atrophic nails as well as hyperpigmentation on the hands. Pertinent initial laboratory findings showed hypoproteinemia (4.1 g/dl) and hypokalemia $(1.7 \mathrm{mmol} / \mathrm{l})$. In addition, serological examination for anti-H. pylori IgG antibody was positive. Colonoscopy revealed an advanced rectal villous adenocarcinoma (stage II) in the background of diffuse typical feature of CCS polyps throughout the colorectum, as recently reported [5]. Upper endoscopy also showed 
numerous reddish polypoid lesions within the stomach. In this diffuse polyposis, a 7-mm discolored polyp, which was slightly larger than the surrounding CCS polyps, was identified at the greater curvature of the lower body (Fig. 1A). A well-defined, flat, elevated lesion was clearly detected by chromoendoscopy (Fig. 1B). Magnified narrow band imaging (NBI) revealed that the microvessels of the tumor surface were irregular, and the diameters and distribution of the vessels were heterogeneous (Fig. 1C), showing an irregular microvascular pattern [6]. A biopsy specimen taken from this lesion was diagnosed as well-differentiated tubular adenocarcinoma. In contrast, the microvessels of CCS polyps showed a regularly elongated and network formation (Fig. 1D).

The patient was diagnosed as having CCS with gastric cancer, and we initially tried to perform an endoscopic mucosal resection of this lesion. However, because the surrounding mucosa was thick and fragile, and we could not elevate the lesion sufficiently even though hyaluronic acid was injected into the submucosal layer, we failed to snare it completely. To complete the resection, we used endoscopic submucosal dissection (ESD) with snaring during the final step of this procedure. It was very difficult to maintain visual contact with the lesion during the ESD procedure, and thus we resected a large area including the cancerous area. The resected area was 43 x $36 \mathrm{~mm}$, and complete en bloc resection with tumor-free margins (Fig. 2A) was accomplished histopathologically. The histology revealed a flat, elevated-type, intramucosal well-differentiated tubular adenocarcinoma with partly moderately to poorly differentiated tubular adenocarcinoma measuring 7X6 mm (Fig. 2B). There was no lymphatic or venous infiltration. The surrounding polyps showed severe edema, cystic dilatation of the glands, and infiltration of inflammatory cells, features that are characteristic of CCS polyps (Fig. 2C). We noted that p53 protein expression was diffusely present in only the cancerous area (Fig. 2D). On mucin-histochemical analysis, the cancerous area was 
positive for CD10 (Fig. 2E), but not for MUC5AC (Fig. 2F), MUC6, or MUC2, indicating a complete intestinal phenotype according to the modified version of Yao's classification [7]. Next, we evaluated the microsatellite instability (MSI) in the cancer and the surrounding CCS polyps using the method described in our previous reports.[8,9] Both lesions were found to be

positive for MSI at the locus of BAT25, D2S123, D5S346, and D17S250 among 5 loci investigated (Fig. 3).

\section{Discussion}

Although CCS is considered a benign condition, gastric cancer occurs in $5.1 \%$ of CCS patients [2], thus indicating that CCS is a high-risk condition for gastric malignancy. Since CCS was first described in 1955, several hundred cases of this syndrome have been reported worldwide, with $75 \%$ of these reports coming from Japan [10]. Furthermore, all of the CCS patients who developed gastric cancer came from Japan, though it is not clear why this was the case. One possibility is that, as Karasawa et al. point out [4], cytotoxin-associated gene A (CagA)-positive H. pylori infection may be associated with the development of gastric cancer, although $\operatorname{CagA}$ was not evaluated in the present case. In most CCS cases, it is impossible to detect a small cancer for an indication of endoscopic treatment among a myriad of CCS polyps. Endoscopic and NBI features were not discussed in the previous reports on CCS associated with gastric cancer; the present report is the first to include those features. Gastric cancers developed in CCS patients were a frequently differentiated histological type [4], showing an irregular microvascular pattern on magnified NBI findings [6,11]. A clue to detecting small cancers, therefore, may be to look for the discolored lesion among multiple reddish polyps, as in the present case, and thereafter to observe the irregular vascular pattern by a magnified NBI endoscopy. It remains unclear why this case revealed a discolored polyp, 
since differentiated tubular adenocarcinoma generally shows a slight redness on endoscopy. One possibility is that the cancer actually showed redness but the different shade of red looked like a discolored lesion compared with the reddish surrounding CCS polyps.

Regarding carcinogenesis in CCS polyps, some researchers have speculated that there exists a hyperplastic-adenoma-carcinoma pathway [2,4]. It has been suggested that individuals with MSI have a higher tendency to accumulate genetic alterations that lead to the transformation of normal cells to cancer cells [12]. Until now, there has been no report on MSI in CCS associated with gastric cancer. Interestingly, MSI was detected in both conditions in our patients, i.e., in the CCS polyps and the gastric cancer, thus supporting that hyperplastic CCS polyps may have the potential to develop into gastric cancer. It has been reported that 78.6\% of differentiated adenocarcinomas in hyperplastic polyps had a gastric phenotype [13]. CCS associated with gastric cancer reported by Karasawa et al. also showed a gastric phenotype that was positive for MUC5AC but not for MUC2 or MUC6 [5]. In contrast, our case showed the complete intestinal phenotype that was positive only for CD10. Yao et al. reported that intestinalization frequently occurred in neoplastic components in hyperplastic polyps, but the complete intestinal type in high-grade dysplasia as in our case was observed less often (20\%, 3 of 15) [7]. Further investigations of cellular phenotypes will be required on a larger sample size with CCS associated with gastric cancers to confirm more clearly the differences of carcinogenesis in these two types of polyps, that is, CCS polyps and common gastric hyperplastic polyps.

To our knowledge, this is the first reported case of CCS with gastric cancer treated endoscopically. Diagnostic points for detecting small cancers in CCS patients may be to look for the discolored polyp among the reddish diffuse polyps by conventional endoscopy, followed by observing the irregular microvessels on the tumor with magnifying NBI 
endoscopy. As patients with CCS polyps are considered to be at high risk for gastric cancer from the viewpoint of genetic alterations, careful follow-up studies are necessary for the early detection of GI malignancies.

\section{Acknowledgements}

The authors would like to thank Ms. Noriko Kamiya and Ms. Masayo Togawa for valuable help in preparing the slide sections and immunostaining. 


\section{References}

1. Cronkhite LW Jr, Canada WJ. Generalized gastrointestinal polyposis: An unusual syndrome of polyposis, pigmentation, alopecia, and onychodystrophy. $N$ Engl J Med 1955;252:1011-5.

2. Egawa T, Kubota T, Otani Y et al. Surgically treated Cronkhite-Canada syndrome associated with gastric cancer. Gastric Cancer 2000;3:156-60.

3. Yamaguchi K, Ogata Y, Akagi Y et al. Cronkhite-Canada syndrome associated with advanced rectal cancer treated by a subtotal colectomy: report of a case. Surg Today 2001;31:521-6.

4. Karasawa H, Miura K, Ishida K et al. Cronkhite-Canada syndrome complicated with huge intramucosal gastric cancer. Gastric Cancer 2009;12:113-7.

5. Watari J, Sakurai J, Morita T et al. A case of Cronkhite-Canada syndrome complicated by McKittrick-Wheelock syndrome associated with advanced villous adenocarcinoma. Gastrointest Endosc 2010 Sep 29. [Epub ahead of print].

6. Yao K, Anagnostopoulos GK, Ragunath K. Magnifying endoscopy for diagnosing and delineating early gastric cancer. Endoscopy 2009;41:462-7.

7. Yao T, Kajiwara M, Kuroiwa S et al. Malignant transformation of gastric hyperplastic polyps: alteration of phenotypes, proliferative activity, and p53 expression. Hum Pathol 2002;33:1016-22.

8. Watari J, Moriichi K, Tanabe H et al. Differences in genetic instability and cellular phenotype among Barrett's, cardiac, and gastric intestinal metaplasia in a Japanese population with Helicobacter pylori. Histopathology 2009;55:261-9.

9. Moriichi K, Watari J, Das KM et al. Effects of Helicobacter pylori infection on genetic instability, the aberrant $\mathrm{CpG}$ island methylation status and the cellular phenotype in 
Barrett's esophagus in a Japanese population. Int J Cancer 2009;124:1263-9.

10. Ward EM, Wolfsen HC. Review article: the non-inherited gastrointestinal polyposis syndromes. Aliment Pharmacol Ther 2002;16:333-42.

11. Nakayoshi T, Tajiri H, Matsuda K et al. Magnifying endoscopy combined with narrow band imaging system for early gastric cancer: correlation of vascular pattern with histopathology (including video). Endoscopy 2004;36:1080-4.

12. Lee HS, Lee BL, Kim SH et al. Microsatellite instability in synchronous gastric carcinomas. Int J Cancer 2001;91:619-24.

13. Kushima R, Hattori T. Histogenesis and characteristics of gastric-type adenocarcinomas in the stomach. J Cancer Res Clin Oncol 1993;120:103-11. 


\section{Figure legends}

Figure 1. A, Upper endoscopy showed a discolored polyp (arrow) surrounded by tiny CCS polyps. B, With chromoendoscopy, the lesion was more clearly revealed as a well-defined, flat, elevated lesion that is slightly larger than the surrounding CCS polyps. C, Magnified NBI revealed an irregular microvascular pattern on the tumor. D, Meanwhile, the microvessels of CCS polyps developed a regularly elongated and network formation.

Figure 2. A, Resected specimen revealed multiple polyps and a small tumor measuring 7X6 mm (arrowhead). B, The histology showed intramucosal well-differentiated tubular adenocarcinoma with partly moderately to poorly differentiated adenocarcinoma. C, The histology of the surrounding mucosa showed hyperplasia with stromal edema, cystic dilatation of the glands, and infiltration of inflammatory cells, which are characteristic of CCS polyps. D, The overexpression of p53 protein was seen in the cancerous area. E, The cancer was positive for CD10 but not for MUC5AC (F).

Figure 3. Microsatellite instabilities on D2S123 and D5S346 were seen as the appearance of some additional alleles (arrows) in both the CCS polyp and the gastric cancer compared with the control. Control DNA was extracted from the muscularis propria of the resected colon (Reference 5). 


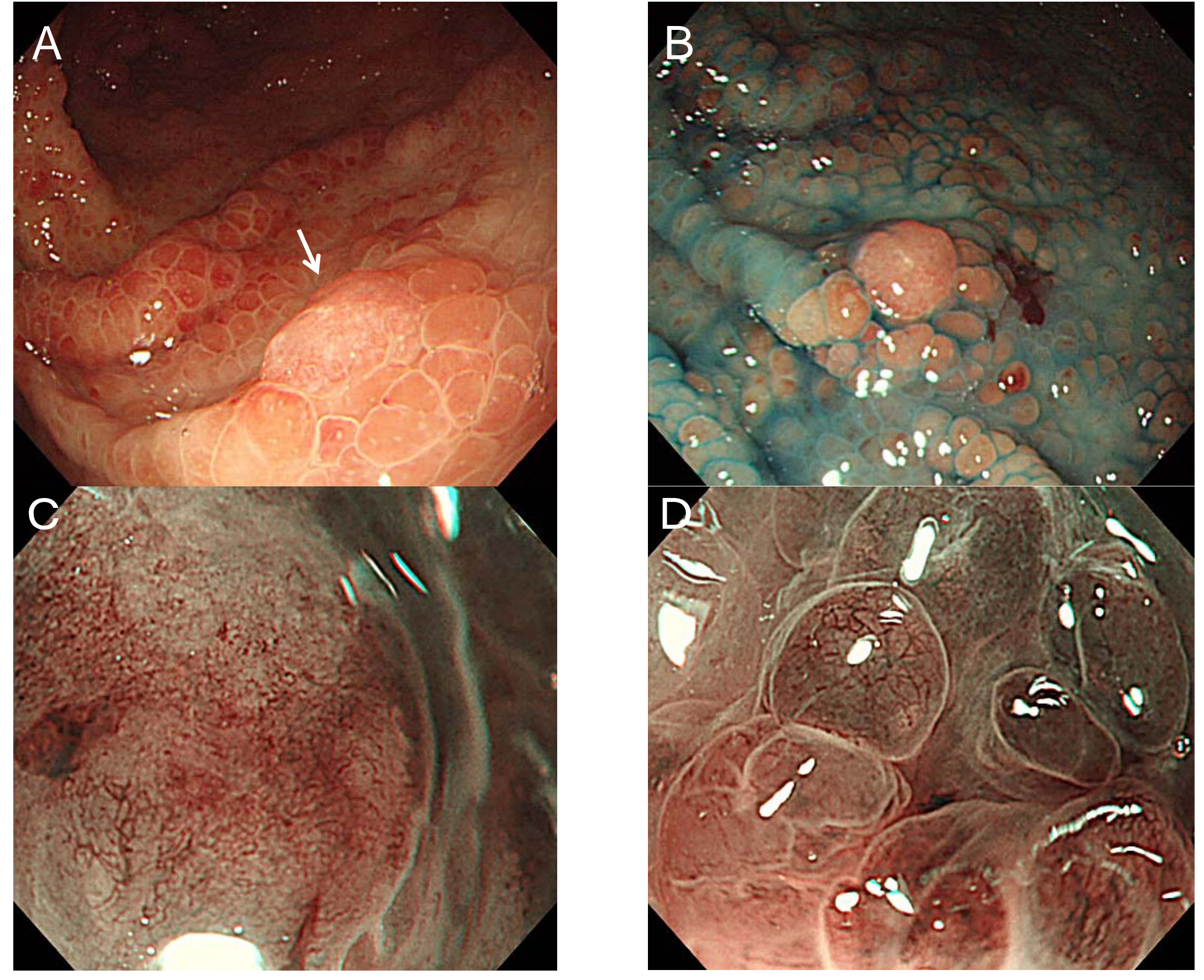

Fig. 1 
A

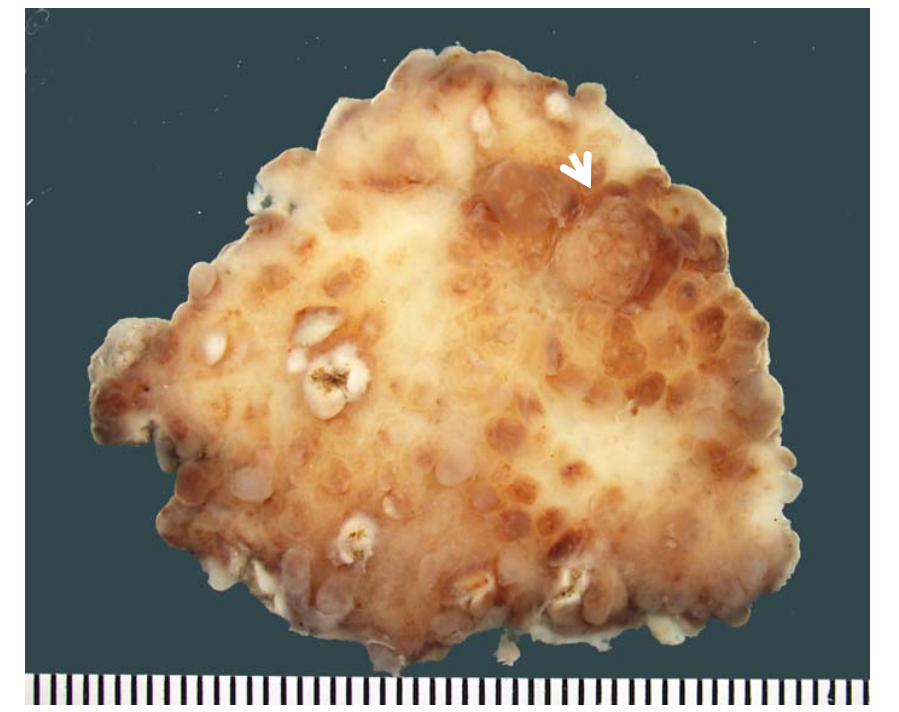

D

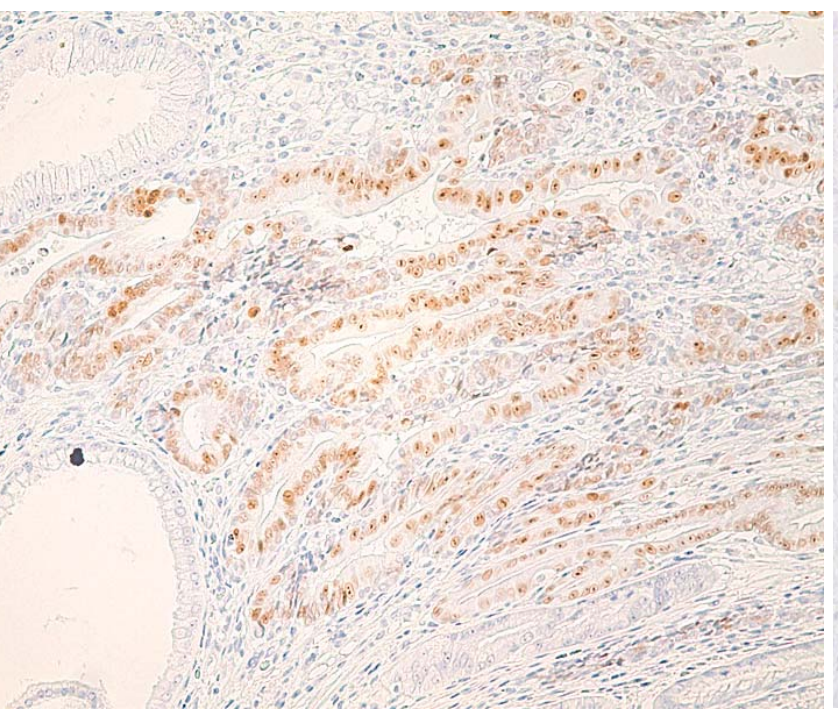

B

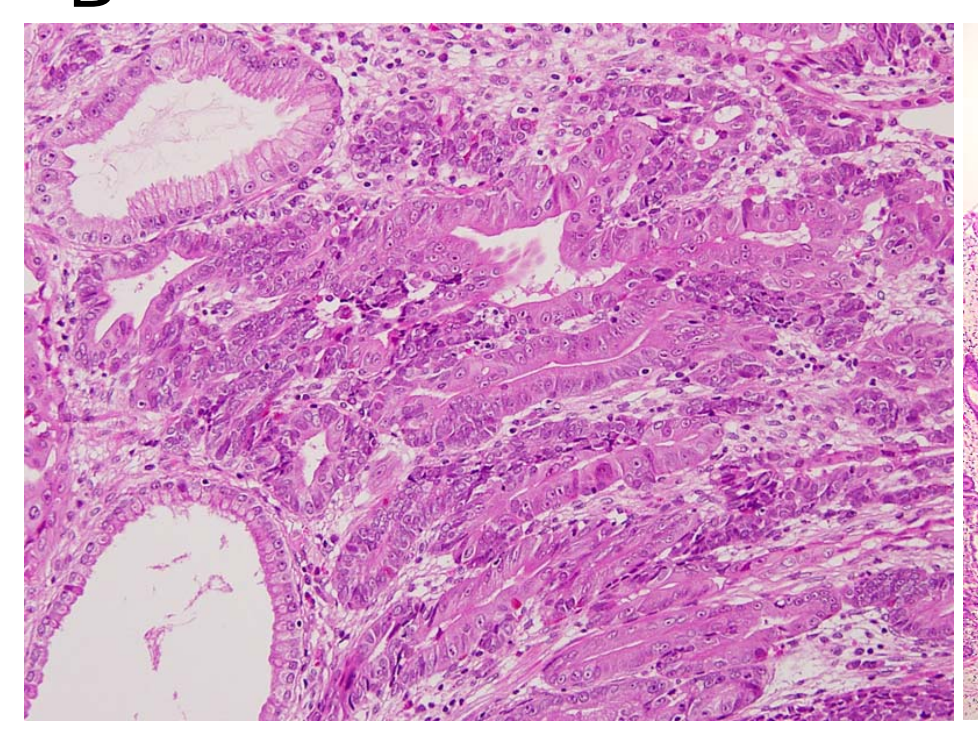

E

Fig. 2
C
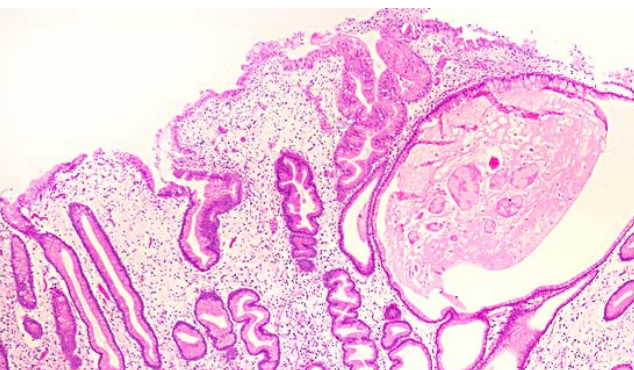

$\int_{00}^{3} 08^{3}=$ 00,0000

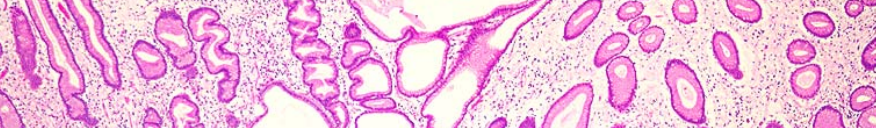
C.5. 1.1.1.

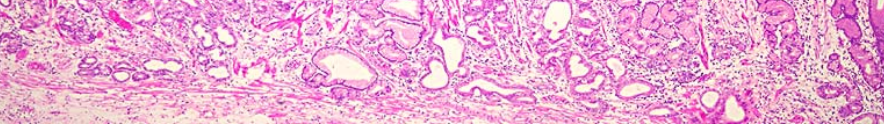

F

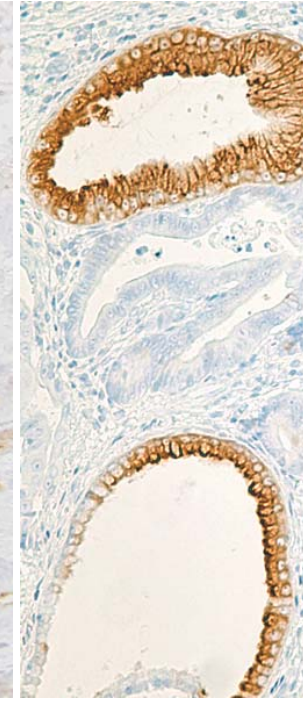

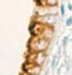




\section{D2S123}

Control

CCS polyp

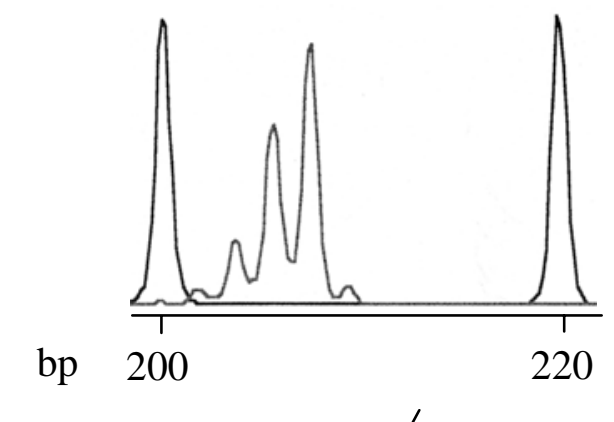$$
\text { bp }
$$

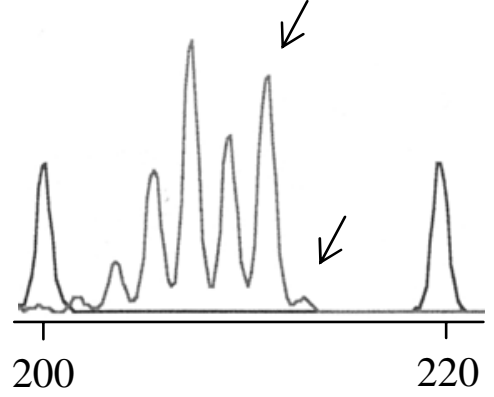

Gastric cancer

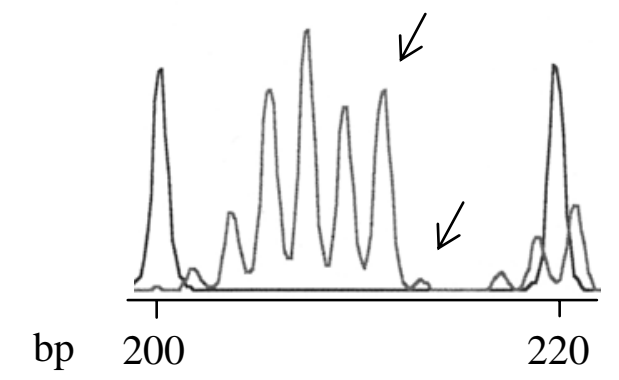

D5S346

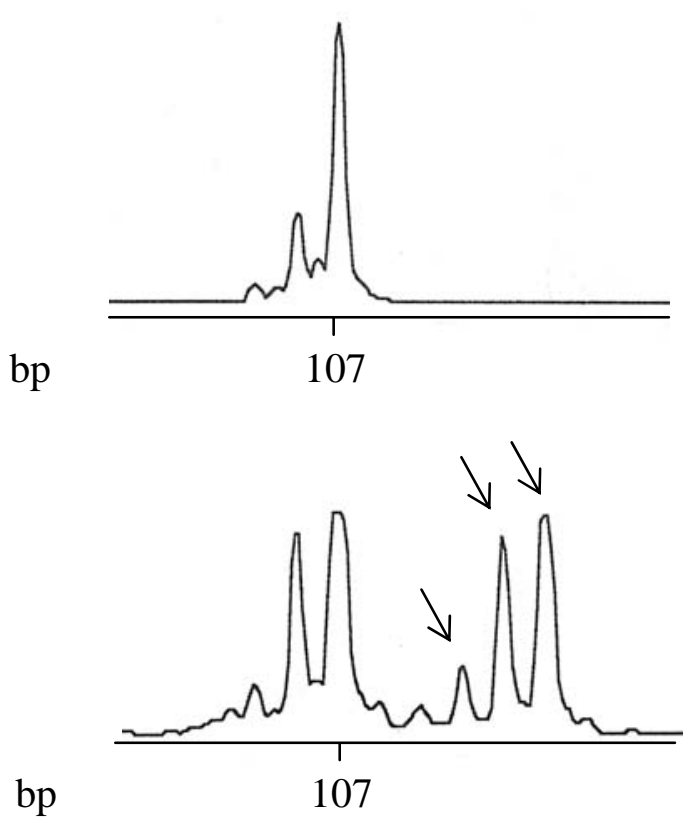

bp

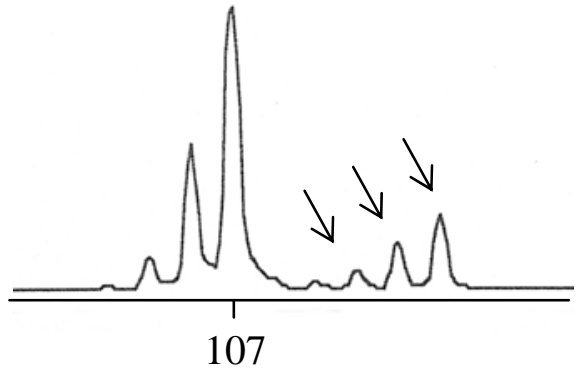

Fig. 3 